\title{
Frequency regulation of modern power system using novel hybrid DE-DA algorithm
}

\author{
Sayantan Sinha ${ }^{1}$, Ranjan Kumar Mallick ${ }^{2}$ \\ ${ }^{1}$ Department of Electrical Engineering, Siksha 'O' Anusandhan Deemed to be University, India \\ ${ }^{2}$ Department of Elelctrical and Electronics Engineering, Siksha 'O' Anusandhan Deemed to be University, India
}

\begin{tabular}{l} 
Article Info \\
\hline Article history: \\
Received Aug 30, 2018 \\
Revised Mar 20, 2019 \\
Accepted Apr 20, 2019 \\
\hline
\end{tabular}

\section{Keywords:}

AGC

Benchmark functions

DE DA hybrid

Deregulated

TID

\begin{abstract}
An attempt has been made to regulate the frequency of an interconnected modern power system using automatic generation control under a restructured market scenario. The system model considered consists of a thermal generation plant coupled with a gas turbine plant in both areas. The presence of deregulated market scenario in an interconnected power system makes it too vulnerable to small load disturbance giving rise to frequency and tie line power imbalances. An attempt has been made to introduce a novel Tilted Integral derivative controller to minimize the frequency and tie line power deviations and restrict them to scheduled values. A maiden attempt has been made to tune the controller gains with the help of a novel hybrid optimization scheme which includes the amalgamation of the exploitative nature of the Differential evolution technique and the explorative attributes of the Dragonfly Algorithm. This hybrid technique is therefore coined as Differential evolution- dragonfly algorithm (DE-DA) technique. Use of some standard benchmark fucntions are made to prove the efficacy of the proposed scheme in tunig the controller gains. The supremacy of the proposed TID controller is examined under two individual market scenarios and under the effect of a step load disturbance. The robustness of the controller in minimizing frequency deviations in the systems is broadly showcased. The superiority of the controller is also proved by comparing it with pre published results.
\end{abstract}

Copyright (C) 2019 Institute of Advanced Engineering and Science. All rights reserved.

\section{Corresponding Author:}

Sayantan Sinha, Department of Electrical Engineering, Siksha 'O' Anusandhan Deemed to be University, Khandagiri Marg, 2, Sum Hospital Rd, Bhubaneswar, Odisha 751030, India.

Email: sayantansinha51@gmail.com

\section{INTRODUCTION}

In recent days, the power system is in astate of transition from centralised control to restructured market scenario. The restructured market consists of GENCOs (Generation companies) DISCOs (Distribution companies), TRANSCOs (Transmission companies) and a control operator named as Independent Service Operator (ISO). The coordinated control of a power system of such a huge scale is tedious and much more complicated. The prime facie objective of AGC in an interconnecped power system is to ensure that the deviations in frequency and the power flow in the tie-lines are restricted within nominal values. In a deregulated market scenario, the ISO plays an ancillary role in ensuring the stable operation of the power system [1,2]. The main role of Automatic Generation control is to maintain an equity between the load demands and the generation of each area. It also pays a keen attention to the fact that the frequency deviation and the tie line power variations should stay within specified limits [3].

In the recent years many researches have been made based on the performance of automatic generation control in the power system world like analysis of the power flow taking AGC into consideration in multi-area interconnected power grid taking into consideration the deregulated market scenario [4]. 
Paper [5] deals with the Utilisation of ultra-capacitor in load frequency control under restructured STPPthermal power systems using WOA optimised PIDN-FOPD controller. Paper [6] concentrates on automatic generation control of two-area hydro-thermal system considering governor dead band under deregulated environment. In paper [7], a work has been done on an algorithm technique known as symbiotic organisms search for AGC in the interlinked power environment which includes wind farms. A recent paper [8] on AGC describes multiple unit of multiple area of deregulated power network by considering an algorithm method known as novel-quasi oppositional harmony search. A research has been made on the judgment of the influence of unreliability on AGC systems [9]. A method of modeling technique and a survey has been made on system stability of AGC on radio systems in smart grids [10]. Paper [11] describes the safety games for threat minimization in automatic generation control. In previous years, a work has been done on a model based strike diagnosis and reduction for automatic generation control [12]. From the study of literature, it was found that the activity of the whole power system network depends on various optimization techniques, structure of the controller. So, the innovations of various optimization techniques are always a welcoming step for the role enhancement of automatic generation control of the entire power system. Many works have been done on various algorithm techniques and methods such as a flower pollination technique based AGC of interlinked power environment and cross characterized 'gbest'-mentored gravitational search and marking search algorithm for AGC of multiple area power system [13, 14]. Several strategies have been made for control for automatic generation control in multi terminal DC grids [15] and other strategies such as in [16], divided model predictive control strategies with demand to power system automatic generation control and in paper [17], distributed automatic generation control using horizontal-based method for large perforation in generation of wind. In [18], an approach has been made to AGC with different nonlinearities by using two degrees of PID controller. Paper [19] brings to light the implementation of an ANFIS based controller for the Load frequency studies in deregulated market. Load and frequency control for an interconnected power system using fractional order controllers were effectively discussed in [20].

In this work an attempt has been made to develop a novel controller with the gains tuned by a hybrid optimization technique. The main objectives of the proposed work are: design of a novel tilted integral derivative controller for the AGC of a multi-source power system under deregulated environment as proposed in [21]; optimization of the gains of the proposed controller with a hybrid DE-DA technique and comparison with the previously published results [21]; testing of the hybrid technique in some standard benchmark functions and establishing the superiority of the optimization scheme; analysis of the system dynamic parameters and comparison with the pre published results [21] and to establish the superiority of the proposed DE-DA optimized TID controller over DE optimized PID controller.

\section{SYSTEM CONSIDERED}

The system proposed in this work is a two area system having equal power ratings $2000 \mathrm{MW}$ each. The linearized transfer function model of the system is clearly depicted in Figure 1. Each area is inclusive of a thermal unit and a gas generating unit. The thermal unit is coupled with a reheat turbine in order to increase its efficiency. The system parameters are listed down in Appendix A. Due to the presence of more than one GENCO in the system, the apf (ACE participation factor) is to be effectively chosen. APF stands for those coefficients that distribute the ACE (Area control error) among the GENCOs.

This paper incorporates the concept of a mutual contract that exists between the Distribution companies (DISCOs) and the generation companies (GENCOs) of an interconnected power system. This mutual contract can exist in various combinations and is well explained mathematically by the Distribution Participation Matrix (DPM). In a DPM, the GENCOs are denoted by the rows and the DISCOs are labeled in the columns. Every individual entry in the DPM gives us a picture of the fraction of the load that is in the contract between the DISOC and the corresponding GENCO.

The actual tie line power flow can be mathematically expressed as

$$
\Delta \mathrm{P}_{\text {tieactual }}=\frac{2 \pi \mathrm{T}_{12}}{\mathrm{~s}}\left(\Delta \mathrm{f}_{1}-\Delta \mathrm{f}_{2}\right)
$$

The tie line power flow in a deregulated environment can be explained as:

$$
\begin{aligned}
\Delta P_{\text {tiescheduled }} & =\sum_{i=1}^{2} \sum_{j=3}^{4} c p f_{i j} \Delta P_{L j}-\sum_{i=3}^{4} \sum_{j=1}^{2} c p f_{i j} \Delta P_{L j} \\
\Delta P_{\text {tiescheduled }} & =\left[P_{\text {expl }}\right]-\left[P_{\text {impl }}\right]
\end{aligned}
$$


where $\left\lfloor\mathrm{P}_{\text {exp }}\right\rfloor$ and $\left[\mathrm{P}_{\text {imp }}\right\rfloor$ stands for the net power expected to flow from area 1 according to the DISCO demands and the power actually supplied to the DISCOs respectively. This gives rise to a deviation in tie line power which is mathematically expressed as:

$$
\Delta P_{\text {tieerror }}=\Delta P_{\text {tieactual }}-\Delta P_{\text {tieschedule }}
$$

The area control error in deregulated scheme can me expressed as:

$$
\begin{aligned}
& A C E_{1}=\beta_{1} \Delta f_{1}+\Delta P_{\text {tieerror } 1} \\
& A C E_{1}=\beta_{2} \Delta f_{2}+\alpha_{12} \Delta P_{\text {tieerror } 2}
\end{aligned}
$$

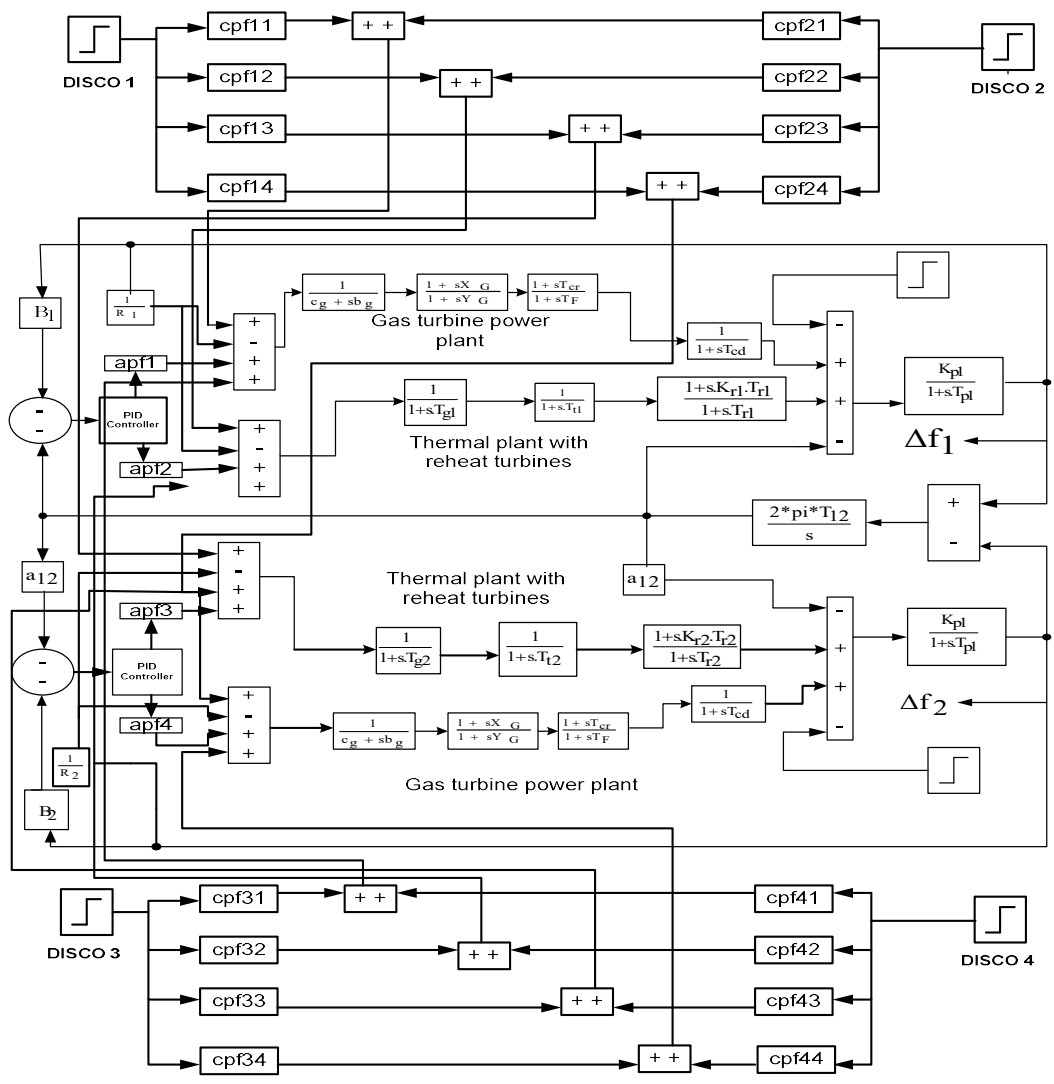

Figure 1. Linearised transfer function model of the system [18]

Where the area coefficient is labeled by $\alpha_{12} \cdot \beta_{1}$ And $\beta_{2}$ stands out as the frequency bias constants of the respective areas. In the proposed system there are more than one GENCOs and so the Area Control Error needs to be shared between all the GENCOs proportional to the contributions for AGC.

\section{CONTROLLER STRUCTURE}

The Proportional Integral Derivative (PID) controller is the most trusted feedback controller used so far. The PID controller accepts an error value as its input. The error is basically the difference between the system variable to be controller and a standard reference point. The control action is exercised using three gain parameters:

- $\quad$ Proportional term [P]: mainly governed by error occurring at time $\mathrm{t}$

- Integral term [I]: governed by the summation of all past error

- $\quad$ Derivative term [D]: involved in predicting errors occurring at $\mathrm{t}+1$.

Frequency regulation of modern power system using novel hybrid DE-DA algorithm (Sayantan Sinha) 
The control action of a PID control can be mathematically represented as:

$$
\mathrm{Y}(\mathrm{s})=K_{p}+s K_{d}+\frac{K_{i}}{s}
$$

Where $K_{p}$ stands for the proportional gain, $K_{i}$ stands for the integral gain and $K_{d}$ denotes the differential gain. This PID is a low order system but has its applications in broad areas. This type of controller mainly finds its application in SISO( single input single output) system. MIMO (Multi input multi output) systems basically are divided into a number of SISO loops and a PID controller is employed for individual of them. The robustness of the PID controller is the main reason for its wide spread acceptance in industrial control. However for an optimum operation of the PID controller, the selection of the above mentioned gains are of prime concern.

\subsection{Tilted integral derivative controller}

The main objective of a Tilted Integral Derivative controller is to provide a feedback action as effective as the PID controller but with the results very close to the theoretical response. The TID controller has the proportional compensator of the PID controller replaced using a 'tilt' compensation. Mathematically the 'tilt' can be expressed by $s^{\frac{1}{n}}$. The tilt compensator mainly generates a frequency dependent feedback gain with a tilt or a shape inclination towards the theoretical or conventional compensation value. As a result the compensation came to be termed as Tilted Integral Derivative controller. The value of ' $n$ ' for the tilt function is between 2 and 3. When compared to the conventional PID controller, the coefficients of the respective transfer functions mainly has a value of $0,+1$ or -1 . On contrary the frequency coefficient in case of tilted controller has a coefficient of $(1 / \mathrm{n})$. This effectively helps to improve the compensating action of the controller. The schematic diagram of the Tilted integral derivative controller is given in Figure 2.

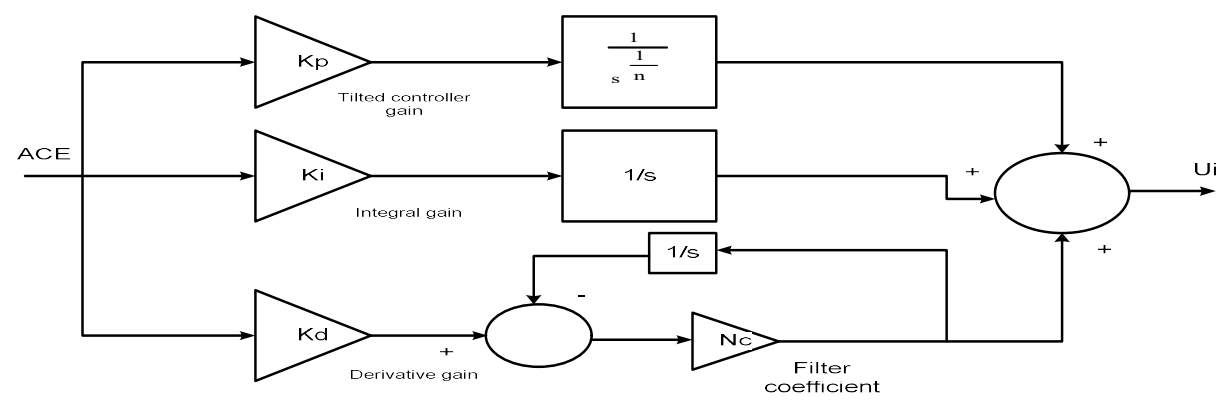

Figure 2. The tilted integral derivative controller

\section{OPTIMIZATION TECHNIQUE PROPOSED}

\subsection{Differential evolution}

First coined by storn and price [22], the differential evolution is mainly known for its simplicity, efficacy and a tremendous high sense of reliability. It is by principle different from genetic algorithm because the technique of Genetic Algorithm still relies on crossover whereas Differential evolution mainly focuses on mutation process for the generation of new solutions.

The successive difference between the two solutions of a randomly generated population mainly brings about the process of mutation. The optimization technique starts with the generation of a random population of size N. Each and every member of the population is to be maintained within limits. The workflow of the optimization technique mainly includes three processes namely mutation, crossover and selection. In this optimization scheme, two randomly generated populations are taken into considerationi.e the old population and the new population in which the members are generated from the old one by the process of mutation and crossover.

The cross over operation done by mixing the mutant vector parameters with the target vector gives rise to a trial vector. The target vector in the next generation is substituted by the trial vector. The evolutionary pathway of DE is shown below:

Int. J. of Adv. in Appl. Sci. Vol. 8, No. 2, June 2019: 103 - 116 
a. Initialization of a random population of size NP consisting of real parameter vectors of size D. If the upper bound of the population is $X_{j}^{U}$ and the lower bound is $X_{J}^{L}$ then it is to be ensured that the randomly generated populations stay between the limits $\left[X_{J}^{U}, X_{J}^{L}\right]$.

b. The mutation process usuallydeals with a vector from the old population to act as the target vector for the new generation. The mutation process is mainly done by calculated the weighted difference between two randomly generated vectors and adding it to another randomly generated vector. The process can be mathematically expressed as:

$$
V_{i, G+1}=X_{r 1, G}+F C\left(X_{r 1, G}-X_{r 2, G}\right)
$$

Where FC is a constant which varies from $(0,2)$.

c. The main advantage of henew vector generated by mutation is that it increases the diversity of the search space by a large extent. The crossover operation usually needs the addition of the mutant vector to the target vector and formation of a new vector. It basically involves three parents for the formation of their offspring's. Mathematically it can be expressed as:

$$
U_{j, i, G+1}=\left\{\begin{array}{l}
V_{j, i, G+1} \text { ifrand }_{j, i} \leq C \text { Rorj }=I_{\text {rand }} \\
X_{j, i, G+1} \text { ifrand }_{j, i}>C \text { Rorj } \neq I_{\text {rand }}
\end{array}\right\} .
$$

In order to maintain the population size throughout the entire length of the process, the selection operation is necessary. This operation involves the target vector $\mathrm{X}_{\mathrm{i}, \mathrm{G}}$ to be compared with $\mathrm{V}_{\mathrm{i}, \mathrm{G}+1}$ and the better fitness possessing vector attains entry to the new generation.

\subsection{Dragonfly algorithm}

Brought to picture by Mirjalili [23], the dragonfly algorithm draws its inspiration from the steady streaming activities of the dragonflies. Supposedly small creatures and harming almost all other insects, the most astonishing fact about the Dragonflies are their strictly adhered social behaviour. The reason for swarming behaviour displayed by the Dragonflies is for the purpose of hunting and migration. The process of hunting is discussed as feeding or static swarm and that of migration is termed as dynamic or migratory swarm [24].

These two behavioural traits replicate the two main phases of optimization: exploration and exploitation. These two phases can be expressed as follows:

- Separation: the term implies the tendency of individuals to avoid collision from other nearby individuals in their path of motion.

- Alignment: The term stands for the matching of velocities of one individual with other in the entire population.

- Cohesion: The term implies the tendency of each individual to travel towards the centre part of the group i.e. towards the central solution.

- Attraction towards a food: This term mathematically implies the attractive behaviour of the dragonflies towards any random source of food.

- $\quad$ Distraction from enemy: the term correctly hints at the unique ability of the dragonflies to distract their enemy to a separate course and saving the entire population.

Taking into consideration the above behaviour of the flies, they are mathematically modelled as:

$$
S_{i}=-\sum_{j=1}^{N} X-X_{j}
$$

Where $S_{i}$ is the separation vector and $\mathrm{X}$ is the position of the current individual and $\mathrm{X}_{\mathrm{j}}$ is the position of the $\mathrm{j}^{\text {th }}$ individual.

$$
A_{i}=\frac{\sum_{j=1}^{N} V_{j}}{N}
$$

Frequency regulation of modern power system using novel hybrid DE-DA algorithm (Sayantan Sinha) 
Where $A_{i}$ is the alignment vector and $V_{j}$ is the velocity of the current individual that is to be matched with the rest of the swarm.

$$
C_{i}=\frac{\sum_{j=1}^{N} X_{j}}{N}-X
$$

Where $C_{i}$ is the cohesion vector, $N$ indicates the number of particles, $X_{j}$ indicates the position of the current particle and $\mathrm{X}$ indicates the central solution as discussed above.

$$
F_{i}=X^{+}-X
$$

Where $\mathrm{X}^{+}$and $\mathrm{X}$ denotes the current individual position and that of the source of food respectively.

$$
E_{i}=X^{-}+X
$$

Where $\mathrm{X}^{-}$and $\mathrm{X}$ stands for the position of the current dragonfly and its enemy respectively.

The step vector for the updation of the dragonfly position can be calculated as the summation of all these factors and can be mathematically expressed as:

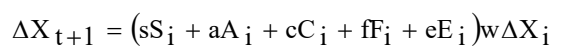

After the generation of the step vector, the position of the dragonflies is updated as:

$$
X_{t+1}=X_{t}+\Delta X_{t+1}
$$

In the absence of any neighboring immediate solution., the dragonflies position is put to updation using a random walk pattern namely called the Levy flight. Thus the position can be mathematically expressed as:

$$
X_{t+1}=X_{t}+\operatorname{Levy}(d) \times X_{t}
$$

Where $\mathrm{d}$ stands for the distance that is to be covered by Levy flight phenomena.

\section{HYBRID OPTIMIZATION SCHEME}

A novel attempt has been made to bring about the amalgamation of the exploitative abilities of an evolutionary computation scheme namely the Differential Evolution and the explorative abilities of a swarm intelligence namely a Dragonfly Algorithm. The technique so developed was coined as Differential Evolution - Dragonfly Algorithm (DE-DA). The entire code has been developed in MATLAb 2013 b platform. Use of some standard unimodal and multimodal benchmark fucntions has been used for testing the efficacy anf the supremacy of the desined novel hybrid DE -DA technique.

In order to prevent any slight bit of anomaly in the comparisons, the function evaluation parameters are considered the same for all the considered fucntions. The hybrid codes are simulated for 30 times and the results were put to statistical analysis. Table 2 summarizes these results. For each method the worst, mean, median, best, and standard deviation from the 30 independent runs were calculated and compared.

In order to establish the robustness of the proposed hybrid technique, the standard deviation and the mean of the fitness values obtained over 30 iterations are calculated and observed. Lesser value of standard deviation indicates the equity that exists in the solutions over 30 runs. This infers that an algorithm with lesser value of standard deviation is more likely to be consistent.

Int. J. of Adv. in Appl. Sci. Vol. 8, No. 2, June 2019: 103 - 116 
Int. J. of Adv. in Appl. Sci.

\begin{tabular}{|c|c|c|c|c|}
\hline $\begin{array}{c}\text { Function } \\
\text { name }\end{array}$ & Test function & $\mathrm{N}$ & $S$ & $\begin{array}{l}\text { fo } \\
\mathrm{pt}\end{array}$ \\
\hline Sphere & $F_{1}(x)=\sum_{i=1}^{n} x_{i}^{2}$ & 30 & {$[-100,100]^{\mathrm{n}}$} & 0 \\
\hline Schwefel & $\mathrm{F}_{2}(\mathrm{x})=\sum_{\mathrm{i}=1}^{\mathrm{n}}\left|\mathrm{x}_{\mathrm{i}}\right|+\prod_{\mathrm{i}=1}^{\mathrm{n}}\left|\mathrm{x}_{\mathrm{i}}\right|$ & 30 & {$[-10,10]^{\mathrm{n}}$} & 0 \\
\hline $\begin{array}{l}\text { Rotated } \\
\text { hyper } \\
\text { ellipsoid }\end{array}$ & $\mathrm{F}_{3}(\mathrm{x})=\sum_{\mathrm{i}=1}^{\mathrm{n}}\left(\sum_{\mathrm{j}=1}^{\mathrm{i}} \mathrm{x}_{\mathrm{j}}\right)^{2}$ & 30 & {$[-100,100]^{\mathrm{n}}$} & 0 \\
\hline Schwefel & $\mathrm{F}_{4}(\mathrm{x})=\max \left\{\left|\mathrm{x}_{\mathrm{i}}\right|, 1 \leq \mathrm{i} \leq \mathrm{x}\right\}$ & 30 & {$[-100,100]^{\mathrm{n}}$} & 0 \\
\hline Step & $\mathrm{F}_{5}(\mathrm{x})=\sum_{\mathrm{i}=1}^{\mathrm{n}}\left(\left[\mathrm{x}_{\mathrm{i}}+0.5\right]\right)^{2}$ & 30 & {$[-100,100]^{\mathrm{n}}$} & 0 \\
\hline Ackley & $\mathrm{F}_{6}(\mathrm{x})=-20 \exp \left(-0.2 \sqrt{\left.\frac{1}{\mathrm{n}} \sum_{\mathrm{i}=1}^{\mathrm{n}} \mathrm{x}_{\mathrm{i}}{ }^{2}\right)}-\exp \left(\frac{1}{\mathrm{n}} \sum_{\mathrm{i}=1}^{\mathrm{n}} \cos 2 \pi \mathrm{x}_{\mathrm{i}}\right)+20+\mathrm{e}\right.$ & 30 & {$[-32,32]^{\mathrm{n}}$} & 0 \\
\hline $\begin{array}{l}\text { Griewank } \\
\text { function }\end{array}$ & $\mathrm{F}_{7}=\sum_{\mathrm{i}=1}^{\mathrm{n}}-\mathrm{x}_{\mathrm{i}} \sin \left(\sqrt{\left|\mathrm{x}_{\mathrm{i}}\right|}\right.$ & 30 & {$[-500,500]^{\mathrm{n}}$} & 0 \\
\hline Penalised & $\begin{array}{l}\mathrm{F}_{8}(\mathrm{x})=\frac{\pi}{\mathrm{n}}\left\{10 \sin ^{2}\left(\pi \mathrm{y}_{\mathrm{i}}\right)+\sum_{\mathrm{i}=1}^{\mathrm{n}}\left(\mathrm{y}_{\mathrm{i}}-1\right)^{2}\left[1+10 \sin ^{2}\left(3 \pi \mathrm{y}_{\mathrm{i}+1}\right)\right]+\sum_{\mathrm{i}=1}^{\mathrm{n}} \mathrm{u}\left(\mathrm{x}_{\mathrm{i}}, 10,100,4\right)\right. \\
\mathrm{y}_{\mathrm{i}}=1+\frac{\mathrm{x}_{\mathrm{i}}+1}{4}\end{array}$ & 30 & {$[-50,50]^{\mathrm{n}}$} & 0 \\
\hline
\end{tabular}

\begin{tabular}{|c|c|c|c|c|c|}
\hline Function name & METHOD & BEST & WORST & MEAN & STD \\
\hline \multirow[t]{3}{*}{$\mathrm{F} 1$} & $\mathrm{DE}$ & 12.5817 & 72.6387 & 35.2792 & 16.5974 \\
\hline & $\mathrm{DA}$ & 18.1680 & 58.8918 & 35.3926 & 10.0163 \\
\hline & DE-DA & 7.5605 & 55.6477 & 30.0650 & 12.9463 \\
\hline \multirow[t]{3}{*}{ F2 } & $\mathrm{DE}$ & $1.95 \mathrm{e}+04$ & $1.07 \mathrm{e}+07$ & $1.92 \mathrm{e}+06$ & $2.311 \mathrm{e}+06$ \\
\hline & $\mathrm{DA}$ & $1.26 \mathrm{e}+04$ & $8.001 \mathrm{e}+07$ & $4.96 \mathrm{e}+06$ & $1.445 \mathrm{e}+07$ \\
\hline & DE-DA & 228.6165 & $1.57 \mathrm{e}+07$ & $9062 \mathrm{e}+05$ & $2.9932 \mathrm{e}+06$ \\
\hline \multirow[t]{3}{*}{ F3 } & $\mathrm{DE}$ & $1.16 \mathrm{e}+3$ & $1.26 \mathrm{e}+04$ & $6.194 \mathrm{e}+03$ & $5.76 \mathrm{e}+03$ \\
\hline & $\mathrm{DA}$ & $1.12 \mathrm{e}+03$ & $1.22 \mathrm{e}+04$ & $4.27 \mathrm{e}+03$ & $4.56 \mathrm{e}+03$ \\
\hline & DE-DA & 47.8718 & $2.19 \mathrm{e}+03$ & 748.8607 & 871.0934 \\
\hline \multirow[t]{3}{*}{$\mathrm{F} 4$} & $\mathrm{DE}$ & 0.2221 & 3.0569 & 0.8643 & 0.6937 \\
\hline & DA & 0.0553 & 10.1392 & 2.2868 & 2.3062 \\
\hline & DE-DA & 0.0248 & 1.4754 & 0.3595 & 0.3832 \\
\hline \multirow[t]{4}{*}{ F5 } & $\mathrm{DE}$ & 45.4651 & 150.5548 & 102.1089 & 25.2523 \\
\hline & DA & 40.8339 & 107.4640 & 71.5784 & 21.2027 \\
\hline & DE-DA & 6.0574 & 41.2643 & 17.1603 & 9.9018 \\
\hline & \multicolumn{5}{|c|}{ MULTIMODAL FUNCTIONS } \\
\hline \multirow[t]{3}{*}{ F6 } & $\overline{\mathrm{DE}}$ & 14.1712 & 20.2920 & 18.6768 & 1.6130 \\
\hline & DA & 6.2233 & 19.9485 & 15.6799 & 3.1122 \\
\hline & DE-DA & 3.2943 & 16.1654 & 9.4173 & 3.3874 \\
\hline \multirow[t]{3}{*}{ F7 } & $\mathrm{DE}$ & 6.4211 & 124.8288 & 49.3711 & 39.0981 \\
\hline & $\mathrm{DA}$ & 3.4524 & 75.4961 & 30.1153 & 20.4420 \\
\hline & DE-DA & 0.6638 & 36.7725 & 6.7834 & 9.1282 \\
\hline \multirow[t]{3}{*}{ F8 } & $\mathrm{DE}$ & 14.0331 & $7.454 \mathrm{e}+7$ & $7.9 \mathrm{e}+6$ & $1.66 \mathrm{e}+5$ \\
\hline & $\mathrm{DA}$ & 3.2985 & $1.3 \mathrm{e}+7$ & $1.3 \mathrm{e}+6$ & $2.7 \mathrm{e}+6$ \\
\hline & DE-DA & 0.1777 & $2 \mathrm{e}+6$ & $1.65 \mathrm{e}+5$ & $4.3 \mathrm{e}+5$ \\
\hline
\end{tabular}

\section{ANALYSIS FOR DE-DA}

The best values, worst values, mean values and standard deviation values for the above mentioned eight benchmark functions in Table 1. Table 2 clearly indicates that the values of the statistical parameters are the least for the DE-DA hybrid technique. The results were obtained for 100 iterations. Added to this the DEDA also accounts for the lowest value of standard deviations among the three techniques. This low value of standard deviation implies that the solutions obtained for the 30 iterations are approximately constant and this is a test for consistency of the proposed optimization scheme. Figure 3, Figure 4, Figure 5, Figure 6, Figure 7, Figure 8, Figure 9, and Figure 10 are showing the convergence curve of DE, DA and DE-DA in 8 functions.

Frequency regulation of modern power system using novel hybrid DE-DA algorithm (Sayantan Sinha) 


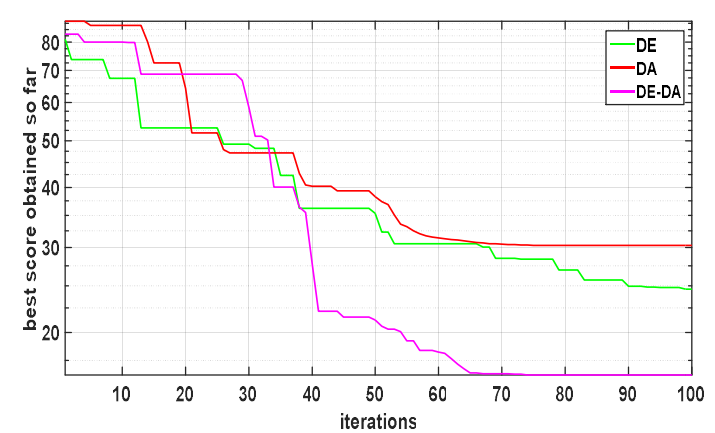

Figure 3. Convergence curve of DE, DA and DE-DA for function 1

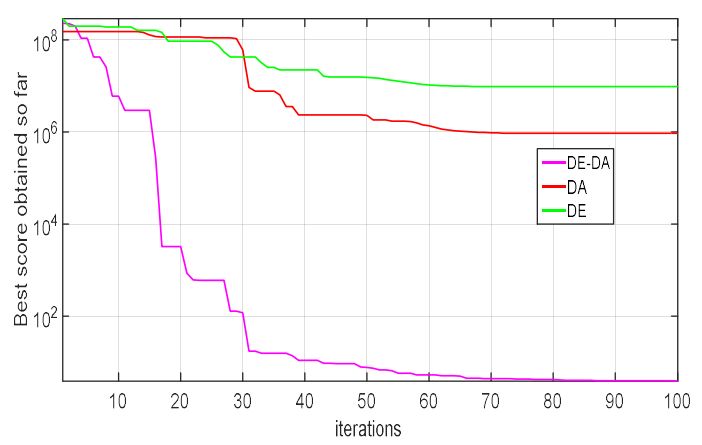

Figure 5. Convergence curve of DE, DA and DE-DA for function 3

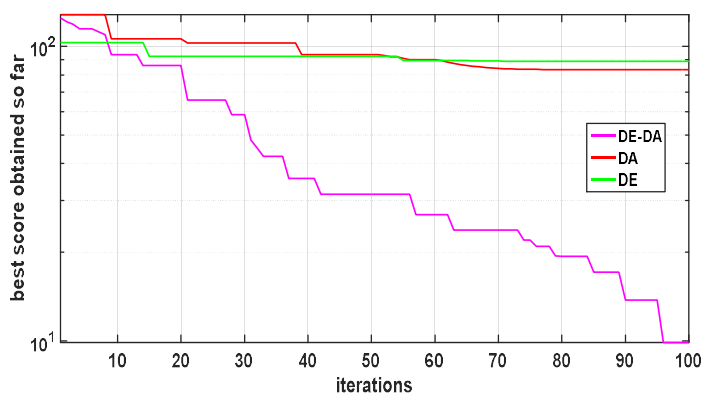

Figure 7. Convergence curve of DE, DA and DE-DA for function 5

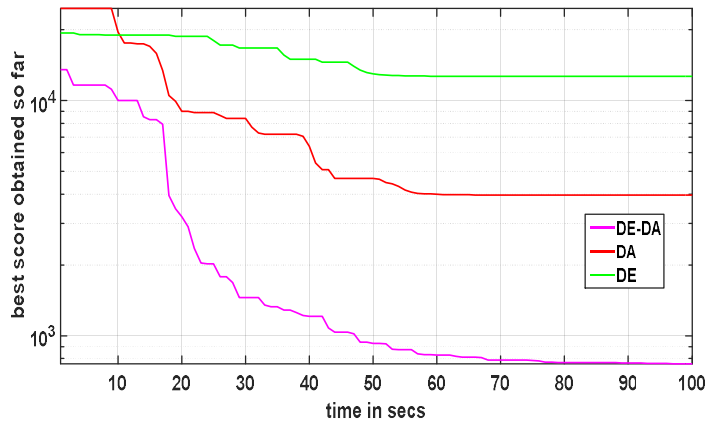

Figure 4. Convergence curve of DE, DA and DE-DA for function 2

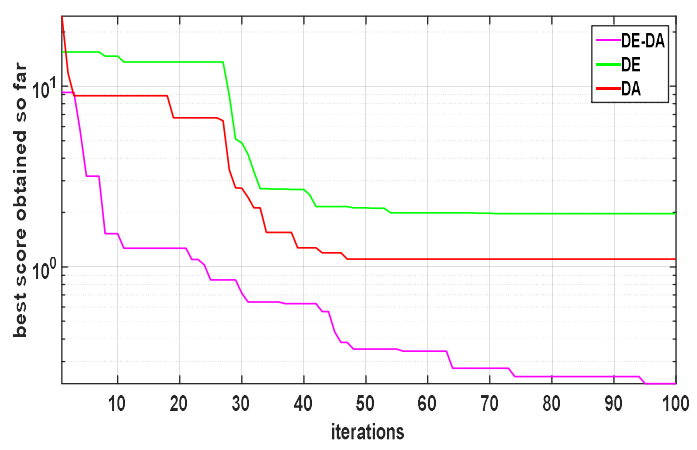

Figure 6. Convergence curve of DE, DA and DE-DA for function 4

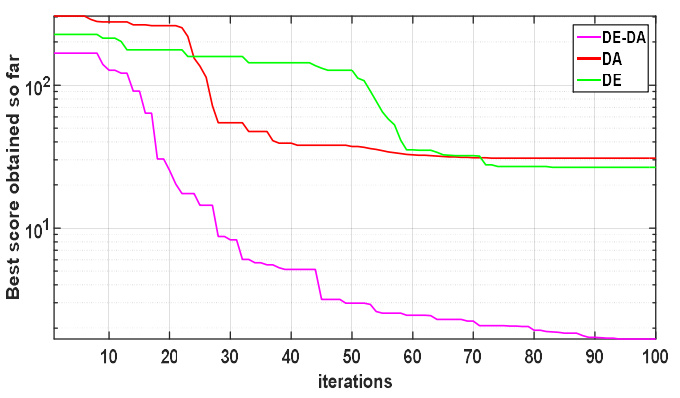

Figure 8. Convergence curve of DE, DA and DE-DA for function 6 


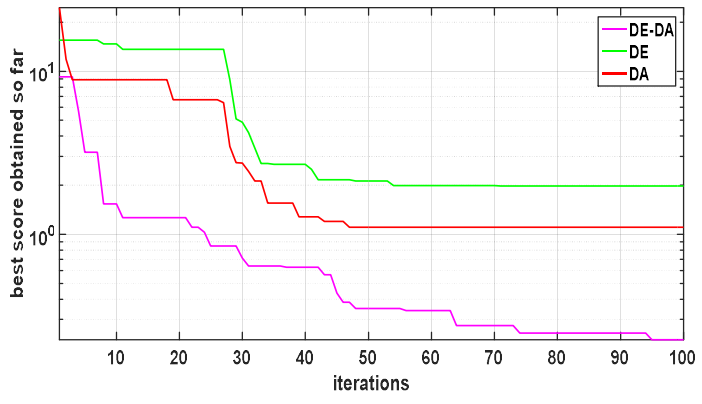

Figure 9. Convergence curve of DE, DA and DE-DA for function 7

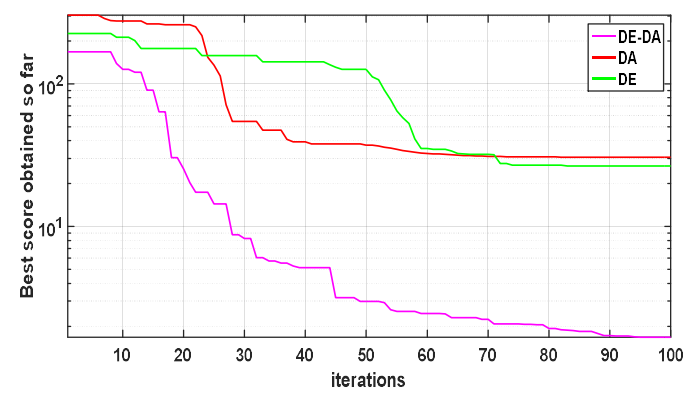

Figure 10. Convergence curve of DE, DA and DE-DA for function 8

\section{APPLICATION OF DE-DA TO AGC PROBLEM}

The proposed hybrid Differential evolution - Dragonfly Algorithm is effectively implemented for obtaining the optimal gain parameters of the Tilted Integral Derivative controller. The hybrid algorithm is said to have a better performance than the De and DA as shown in Table 2. Table 3 lists down the optimal values of the controller gains. The application of the hybrid algorithm to the AGC is governed by the following pseudo code.

Pseudo code for the hybrid optimization:

- Generation of the initial population and specification of the Differential evolution parameters like cross over ratio and mutation frequency.

- $\quad$ Computation of the fitness functions of each of the population members.

- Generation of offsprings using mutation technique.

- Calculation of the fitness function of each generated offspring.

- Selection of the best offspring by cross over technique that includes the parent.

- $\quad$ Replacement of the parents in the total population with the off springs and give rise to a final population.

- $\quad$ The final DE population acts as the initial population for Dragonfly Algorithm.

- Initialization of the step vectors $\Delta \mathrm{X}_{\mathrm{i}}$

- Computation of the fitness values of all the dragonflies.

- $\quad$ Updation of the food source and the enemy position.

- Updation of cohesion vector, separation vector, alignment vector, food distance vector and the enemy attack vector.

- Based on condition of at least one dragonfly in the neighbourhood of the current dragonfly, updation of the position is done.

- $\quad$ Checking of the new particles under boundary conditions.

- $\quad$ Jump over to step 2 until maximum iteration is reached.

Terminate the loop.

\section{RESULTS AND ANALYSIS}

The discussed two area power system model is put to simulation with the help of MATLAB/ Simulink. The tilted integral derivative controller is employed in this case as the secondary controller for minimizing the Area Control Error (ACE) to zero. The gains of the TID controller are set to optimal values with the help of a novel hybrid DE-DA technique. The objective functions employed in the tuning process are as follows:

$$
\begin{aligned}
& \text { IAE }=\int_{0}^{\mathrm{t}}\left(\left|\Delta \mathrm{f}_{1}\right|+\left|\Delta \mathrm{f}_{2}\right|+\left|\Delta \mathrm{p}_{\text {tie }}\right|\right) \mathrm{dt} \\
& \text { ITAE }=\int_{0}^{\mathrm{t}}\left(\left|\Delta \mathrm{f}_{\mathrm{f}}\right|+\left|\Delta \mathrm{f}_{2}\right|+\left|\Delta \mathrm{p}_{\text {tie }}\right| \mathrm{tdt}\right) \\
& \text { ISE }=\int_{0}^{\mathrm{t}}\left(\Delta \mathrm{f}_{1}{ }^{2}+\Delta \mathrm{f}_{2}^{2}+\Delta \mathrm{p}_{\text {tie }}^{2}\right) \mathrm{dt}
\end{aligned}
$$




$$
\operatorname{ITSE}=\int_{0}^{\mathrm{t}}\left(\Delta \mathrm{f}_{1}^{2}+\Delta \mathrm{f}_{2}^{2}+\Delta \mathrm{p}_{\mathrm{tie}}^{2}\right) \mathrm{tdt}
$$

The values of each of the above objective functions during the tuning process is noted and scripted in Table 2. The table clearly indicates that the value of the objective function $\mathrm{J}$ is the minimum in the case of ISE (Integral square error) and henceforth all the tuning process in the paper has been done considering ISE as the cost function.

Table 1. Optimized system parameters by hybrid DE-DA technique

\begin{tabular}{ccccccc}
\hline Market scenario & Kt1 & Kd1 & Ki1 & Kt2 & Kd2 & Ki2 \\
\hline Base case & 0.2401 & 0.2802 & 0.1590 & 0.1539 & 0.0952 & 0.1302 \\
Billateral contract & 0.2556 & 0.1503 & 0.3057 & 0.1995 & 0.1976 & 0.2974 \\
\hline
\end{tabular}

Table 2. Objective function values for base case and bilateral transactions

\begin{tabular}{ccccc}
\hline \multirow{2}{*}{ Market scenario } & \multicolumn{4}{c}{ Objective function } \\
& ITAE & IAE & ITSE & 0.0398 \\
Base case & 1.9137 & 0.5937 & 0.0782 & 0.0409 \\
\hline
\end{tabular}

The analysis is done on two cases:

Case 1: the base case

In this case the GENCOs and DISCOs come into mutual participation for the electricity market over a common area. This implies DISCOs of one area can only come in contract with the GENCOs of the respective areas. This mutual contract scenario can be represented with the help of Distribution Participation Matrix (DPM).

$$
\mathrm{DPM}=\left[\begin{array}{cccc}
0.5 & 0.5 & 0 & 0 \\
0.5 & 0.5 & 0 & 0 \\
0 & 0 & 0 & 0 \\
0 & 0 & 0 & 0
\end{array}\right]
$$

The area participation factor apf is taken equal for all the GENCOs in this particular case. So $\operatorname{apf}_{1}=\operatorname{apf}_{2}=\operatorname{apf}_{3}=\operatorname{apf}_{4}=0.5$

The simulation is carried out inclusive of a SLP of $0.01 \mathrm{pu}$ in area 1 . The demands of DISCOs are generally fixed at $0.1 \mathrm{MW}$. Figure 11, Figure 12 and Figure 13 represents the frequency deviation in area 1 $\left(\Delta \mathrm{f}_{1}\right)$, area 2 frequency deviation $\left(\Delta \mathrm{f}_{2}\right)$ and tie line power flow deviation $\left(\Delta \mathrm{p}_{\text {tie }}\right)$ respectively. Table 3 clearly gives us the values of settling time, maximum overshoot and minimum undershoot for the DE DA optimized TID controller. Effective comparison has been done with the settling time, maximum overshoot and minimum undershoot of DE optimized PID controller when applied to the same physical system under base case. From the tabular comparison it can be easily inferred that the DE-DA tuned TID controller is much more robust and effective than the De tuned PID controller. Figure 14, Figure 15, Figure16 and Figure 17 elaborates the power output pattern of GENCO 1, GENCO 2, GENCO 3 and GENCO 4 respectively.

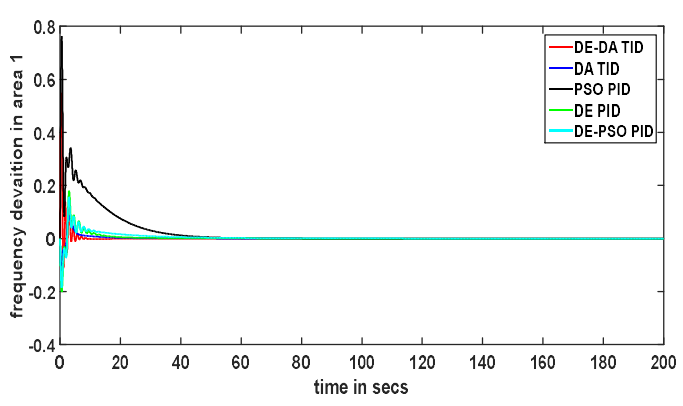

Figure 11. Frequency deviation of area 1

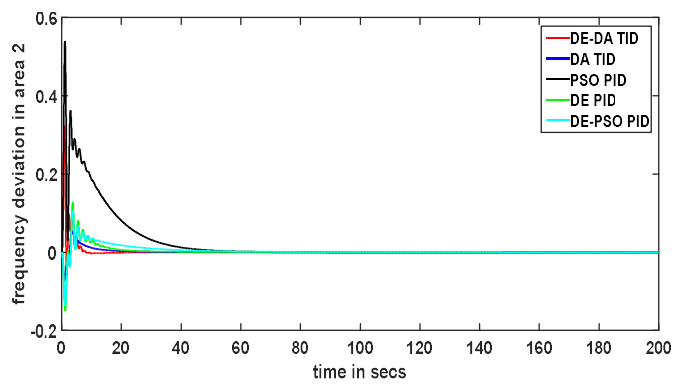

Figure 12. Frequency deviation of area 2

Int. J. of Adv. in Appl. Sci. Vol. 8, No. 2, June 2019: 103 - 116 


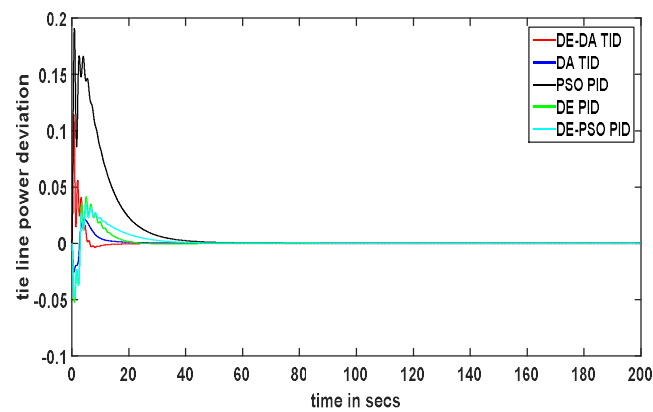

Figure 13. Tie line power deviation

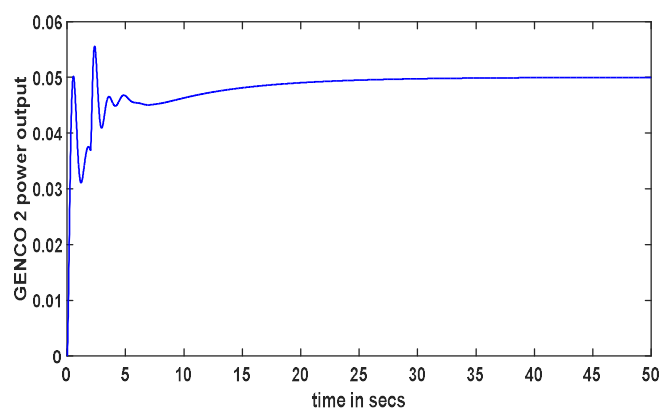

Figure 15. Power supplied by GENCO 2

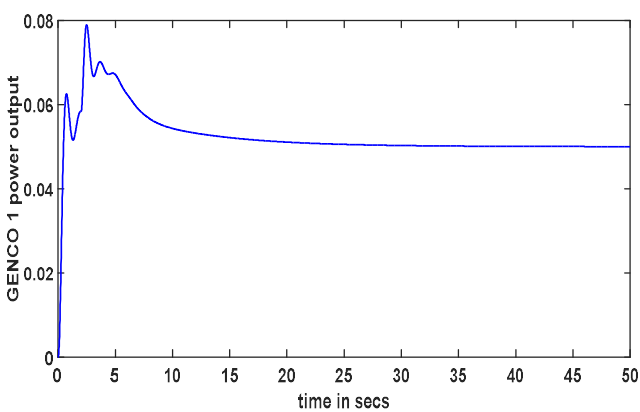

Figure 14. Power supplied by GENCO 1

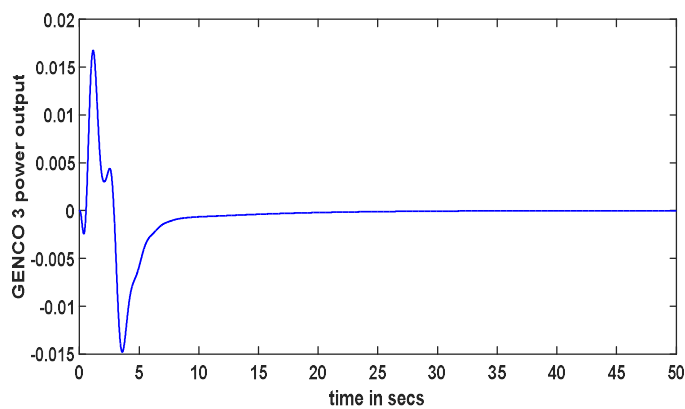

Figure 16. Power supplied by GENCO 3

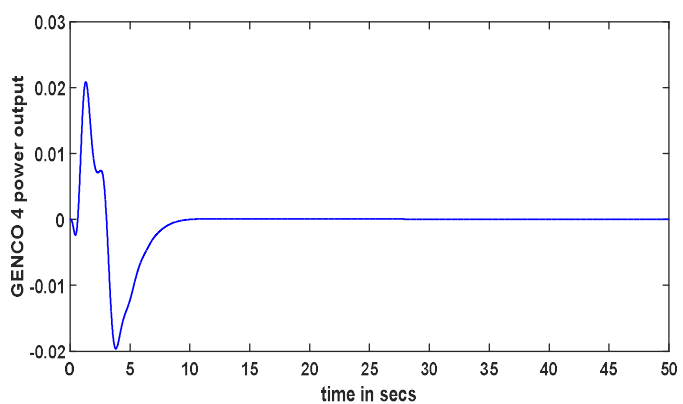

Figure 17. Power supplied by GENCO 4

Case 2: Billateral transaction

In this case the DISOCs and the GENCOs of both area come to a mutual contract participation and the scenario is expressed by the following DPM.

$\mathrm{DPM}=\left[\begin{array}{cccc}0.5 & 0.25 & 0 & 0.3 \\ 0.2 & 0.25 & 0 & 0 \\ 0 & 0.25 & 1 & 0.7 \\ 0.3 & 0.25 & 0 & 0\end{array}\right]$

The area participation factor in this case is taken as reference to [18].

$\operatorname{apf}_{1}=0.75 ; \operatorname{apf}_{2}=1-\operatorname{apf}_{1}=0.25 ; \operatorname{apf}_{3}=\operatorname{apf}_{4}=0.5$. The given system is simulated under bilateral contract scenario subject to a step load perturbation of $0.01 \mathrm{pu}$. Figure 18, Figure 19 and Figure 20 clearly portrays the frequency deviation in area $1\left(\Delta \mathrm{f}_{1}\right)$, area 2 frequency deviation $\left(\Delta \mathrm{f}_{2}\right)$ and tie line power flow deviation $\left(\Delta \mathrm{p}_{\text {tie }}\right)$ respectively. Table 4 lists down the values of the settling time, maximum overshoot and minimum undershoot

Frequency regulation of modern power system using novel hybrid DE-DA algorithm (Sayantan Sinha) 
for the hybrid optimized TID controller and is put to comparison with DE optimized PID controller [18]. Figure 21, Figure 22, Figure 23 and Figure 24 clearly portrays the power output of GENCO 1, GENCO 2, GENCO 3 and GENCO 4 respectively. The comparison clearly indicates the superiority of DE-DA optimized TID controller over DE optimized PID controller under bilateral contract scenario.

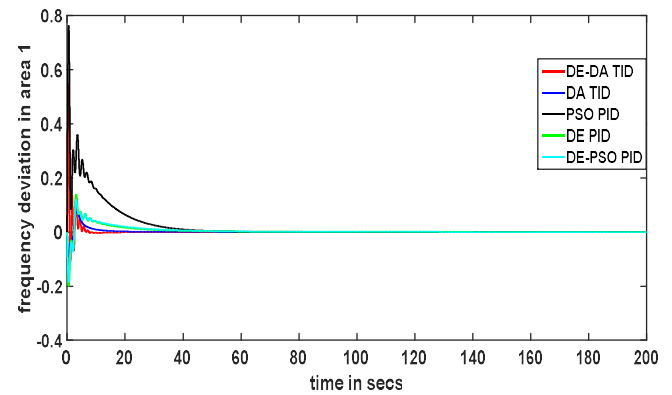

Figure 18. Frequency oscillations in area 1

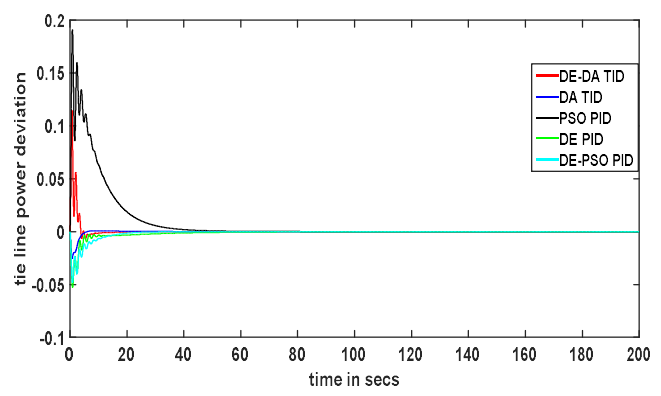

Figure 20. Tie line power variations

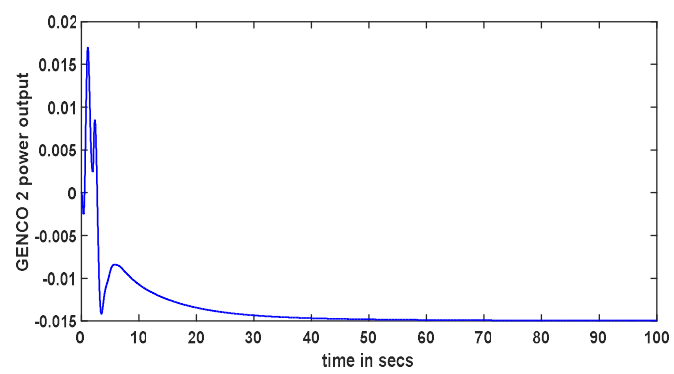

Figure 22. Power supplied by GENCO 2

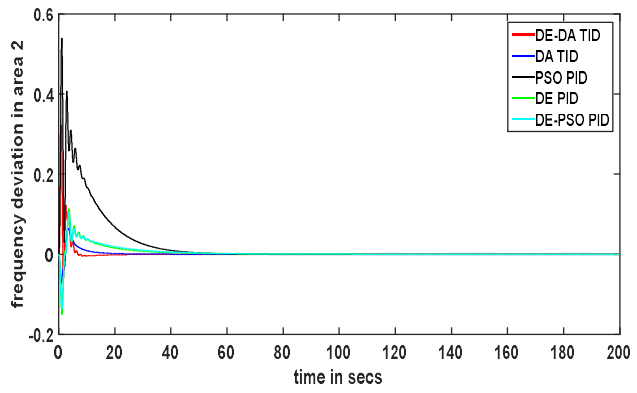

Figure 19. Frequency oscillations in area 2

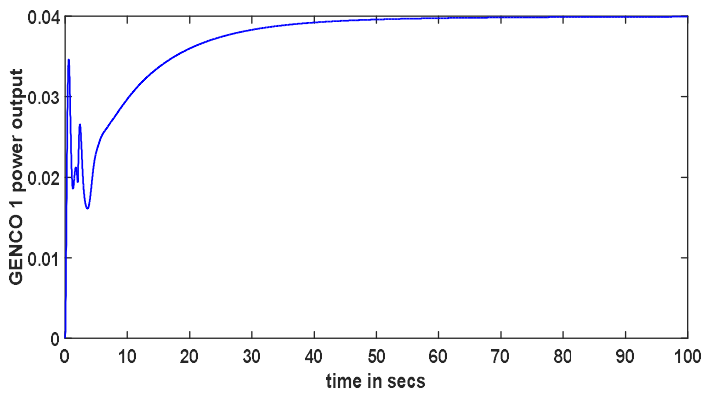

Figure 21. Power supplied by GENCO 1

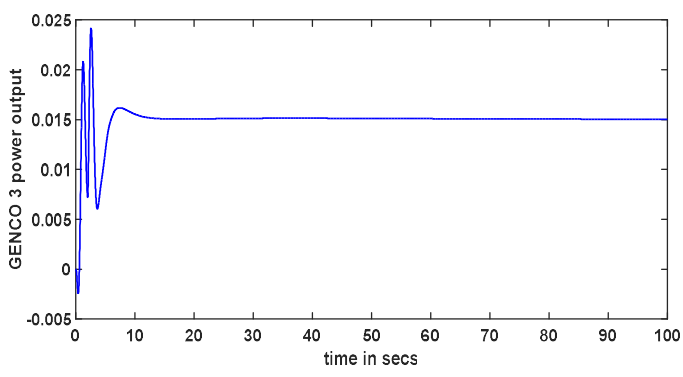

Figure 23. Power supplied by GENCO 3

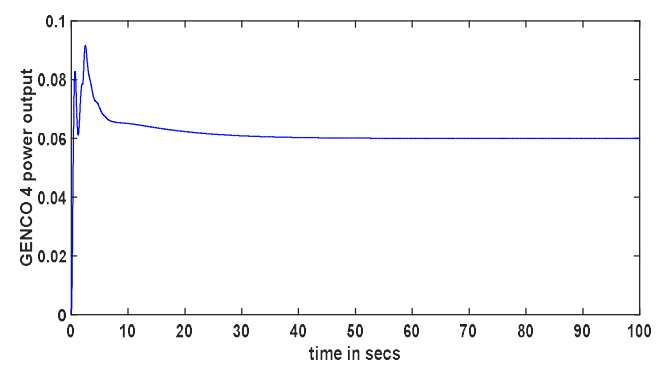

Figure 24. Power supplied by GENCO 4 
Table 3. Dynamic system parameters under base case scenario

\begin{tabular}{ccccc}
\hline Controller type & System data & Settling time & Maximum overshoot & Minimum undershoot \\
\hline \multirow{4}{*}{ PID[18] } & $\Delta \mathrm{f}_{1}$ & 5.52 & 0.0409 & -0.2574 \\
& $\Delta \mathrm{f}_{2}$ & 4.51 & 0.012 & -0.0946 \\
& $\Delta \mathrm{p}_{\text {tie }}$ & 1.88 & 0.0057 & -0.0315 \\
\multirow{2}{*}{ TID } & $\Delta \mathrm{f}_{1}$ & 4.4831 & 0.3500 & -0.0090 \\
& $\Delta \mathrm{f}_{2}$ & 4.420 & 0.9400 & -0.0055 \\
& $\Delta \mathrm{p}_{\text {tie }}$ & 7.0473 & 0.0057 & -0.0315 \\
\hline
\end{tabular}

Table 4. Dynamic system parameters for bilateral scenario

\begin{tabular}{ccccc}
\hline Controller type & System data & Settling time & Maximum overshoot & Minimum undershoot \\
\hline \multirow{4}{*}{ PID[18] } & $\Delta \mathrm{f}_{1}$ & 10.87 & 0.0988 & -0.4664 \\
& $\Delta \mathrm{f}_{2}$ & 11.47 & 0.0683 & -0.2158 \\
& $\Delta \mathrm{p}_{\text {tie }}$ & 3.08 & 0.05 & -0.0185 \\
& $\Delta \mathrm{f}_{1}$ & 7.6598 & 0.1149 & -0.1551 \\
\multirow{2}{*}{ TID } & $\Delta \mathrm{f}_{2}$ & 7.1017 & 0.1096 & -0.0975 \\
& $\Delta \mathrm{p}_{\text {tie }}$ & 9.4850 & 0.0083 & -0.0348 \\
\hline
\end{tabular}

\section{CONCLUSION}

Whenever a modern interconnected power system is considered for study under restructured market scenarios, the power system becomes highly sensible to slight load disturbances. As of concern the maintainence of frequency within scheduled values should be of a major importance. This is effectively attained by the Tilted Integral Derivative controller in the proposed work. Development of a hybrid optimization technique, the DE-DA technique is successfully verified and tested with standard benchmark functions. This technique is thereafter used to provide optimal values to the controller for minimizing the ACE to zero. The hybrid tuned controller has proved to be effective in minimizing frequency as well as tie line power deviations in a shortest time period possible. In order to justify the supremacy of the controller, the results are compared with pre published results. The dynamic performance indices of the system is analysed in both the cases and is listed down in respective tables for clear inference. It can be clearly established that the values of settling time, maximum overshoot and minimum undershoot is considerably less for both market scenarios taken into consideration.

\section{REFERENCES}

[1] Dong, X., Sun, H., Wang, C., Yun, Z., Wang, Y., Zhao, P., Ding, Y., and Wang, Y., "Power Flow Analysis Considering Automatic Generation Control for Multi-Area Interconnection Power Networks," IEEE Transactions on Industry Applications, vol 53(6), pp. 5200-8, 2017.

[2] Kumar, J., Ng, KH., and Sheble, G., "AGC simulator for price-based operation. I. A model," IEEE Transactions on Power Systems, vol. 12(2), pp. 527-32, 1997.

[3] Kumar, J., Ng, KH., and Sheble, G., "AGC simulator for price-based operation. II. Case study results," IEEE Transactions on Power Systems, vol. 12(2), pp. 533-8, 1997.

[4] Sinha, N., Lai, LL., and Rao, VG., "GA optimized PID controllers for automatic generation control of two area reheat thermal systems under deregulated environment," Electric Utility Deregulation and Restructuring and Power Technologies, 2008. DRPT 2008, pp. 1186-1191, 2008.

[5] Saha, A. and Saikia, LC., "Utilisation of ultra-capacitor in load frequency control under restructured STPP-thermal power systems using WOA optimised PIDN-FOPD controller," IET Generation, Transmission \& Distribution, vol. 11(13), pp. 3318-31, 2017

[6] Raju, M., Saikia, LC., Sinha, N., and Saha, D, “Application of antlion optimizer technique in restructured automatic generation control of two-area hydro-thermal system considering governor dead band," Power and Advanced Computing Technologies (i-PACT), pp. 1-6, 2017.

[7] Hasanien, HM. and El-Fergany, AA, "Symbiotic organisms search algorithm for automatic generation control of interconnected power systems including wind farms," IET Generation, Transmission \& Distribution, vol. 11(7), pp. 1692-700, 2016.

[8] Shiva, CK. and Mukherjee, V., "Automatic generation control of multi-unit multi-area deregulated power system using a novel quasi-oppositional harmony search algorithm," IET Generation, Transmission \& Distribution, vol. 9(15), pp. 2398-408, 2015.

Frequency regulation of modern power system using novel hybrid DE-DA algorithm (Sayantan Sinha) 
[9] Apostolopoulou, D., Domínguez-García, AD., and Sauer PW., "An assessment of the impact of uncertainty on automatic generation control systems," IEEE Transactions on Power Systems, vol. 31(4), pp. 2657-65, 2016.

[10] Liu, S., Liu, PX., and El Saddik, A., "Modeling and stability analysis of automatic generation control over cognitive radio networks in smart grids," IEEE Transactions on Systems, Man, and Cybernetics: Systems, vol. 45(2), pp. 223-34, 2015

[11] Law, YW, Alpcan, T., and Palaniswami, M., "Security games for risk minimization in automatic generation control," IEEE Transactions on Power Systems, vol. 30(1), pp. 223-32, 2015.

[12] Sridhar, S. and Govindarasu, M., "Model-based attack detection and mitigation for automatic generation control," IEEE Transactions on Smart Grid, vol. 5(2), pp. 580-91, 2014.

[13] Jagatheesan, K., Anand, B., Samanta, S., Dey, N., Santhi, V., Ashour, AS., and Balas VE, "Application of flower pollination algorithm in load frequency control of multi-area interconnected power system with nonlinearity," Neural Computing and Applications, vol 28(1), 475-88, 2017.

[14] Khadanga, RK. and Kumar, A., "Hybrid adaptive 'gbest'-guided gravitational search and pattern search algorithm for automatic generation control of multi-area power system," IET Generation, Transmission \& Distribution, vol. 11(13), pp. 3257-67, 2016.

[15] McNamara, P., Meere, R., O'Donnell, T., and McLoone, S., "Control strategies for automatic generation control over MTDC grids," Control Engineering Practice, vol. 54, pp. 129-39, 2016.

[16] Venkat, AN., Hiskens, IA., Rawlings, JB. and Wright, SJ., "Distributed MPC strategies with application to power system automatic generation control," IEEE transactions on control systems technology, vol. 16(6), pp. 1192-206, 2008.

[17] Variani, MH. and Tomsovic, K., "Distributed automatic generation control using flatness-based approach for high penetration of wind generation," IEEE Transactions on Power Systems, vol. 28(3), pp. 3002-9, 2013.

[18] Ibrahim, AN., Shafei, MA., and Ibrahim, DK., "Linearized biogeography based optimization tuned PID-P controller for load frequency control of interconnected power system," Power Systems Conference (MEPCON), pp. 1081-1087, 2017.

[19] Selvaraju, RK. and Somaskandan, G., "ACS algorithm tuned ANFIS-based controller for LFC in deregulated environment," Journal of Applied Research and Technology, vol. 15(2), pp. 152-66, 2017.

[20] Gorripotu, TS., Sahu, RK., and Panda, S., "AGC of a multi-area power system under deregulated environment using redox flow batteries and interline power flow controller," Engineering Science and Technology, an International Journal, vol. 18(4), pp. 555-78, 2015.

[21] Hota, PK. and Mohanty B., "Automatic generation control of multi source power generation under deregulated environment," International Journal of Electrical Power and Energy Systems, vol. 75, pp. 205-14, 2016.

[22] Storn, R. and Price, K., "Differential evolution-a simple and efficient heuristic for global optimization over continuous spaces," Journal of global optimization, vol. 11(4), pp. 341-59, 1997

[23] Mirjalili, S., "Dragonfly algorithm: a new meta-heuristic optimization technique for solving single-objective, discrete, and multi-objective problems," Neural Computing and Applications, vol. 27(4), pp. 1053-73, 2016.

[24] KS SR, Murugan, S., "Memory based hybrid dragonfly algorithm for numerical optimization problems," Expert Systems with Applications, vol. 83, pp. 63-78, 2017.

Int. J. of Adv. in Appl. Sci. Vol. 8, No. 2, June 2019: 103 - 116 